\title{
A Rare Case of Right Ventricular Myxoma with Tricuspid Valve Endocarditis
}

\author{
Muhammad Arza Putra, MD, ${ }^{1}$ Jenni Pratita, MD,${ }^{1}$ Jonathan Grantomo, $M D,{ }^{1}$ Budiana Tanurahardja, MD $^{2}$ \\ ${ }^{1}$ Department of Surgery, Faculty of Medicine, Universitas Indonesia / Integrated Cardiovascular Services Unit Cipto \\ Mangunkusumo Hospital, Jakarta Pusat, Indonesia; ${ }^{2}$ Department of Pathology Anatomy, Cipto Mangunkusumo Hospital, Jalan \\ Diponegoro Jakarta, Indonesia
}

\section{ABSTRACT}

Background: Although myxoma is the most common form of benign cardiac tumor, which is a rare condition itself, less than $3-4 \%$ of cases are detected in the right ventricle $(\mathrm{RV})$. The clinical presentations vary widely and are nonspecific, causing challenging diagnosis. Although rare, myxoma can coexist with infective endocarditis (IE).

Case report: We report a rare case of right ventricular myxoma presenting with dyspnea and fever, which was later found to be complicated with concomitant tricuspid valve endocarditis during surgery as well as the performed surgical approach.

Conclusion: RV myxoma is a rare entity requiring a high index of suspicion due to varying nonspecific presentations. Concomitant IE should be suspected in patients with persistent fever. Antibiotics and careful surgical approach are needed to prevent complications, including embolization.

\section{INTRODUCTION}

Cardiac tumors are very rare. Despite being the most common form of benign cardiac tumors, less than 3-4\% of myxomas are detected in the right ventricle (RV). Their varying and nonspecific presentation poses a diagnostic challenge [Koza 2019]. On rare occasions, myxomas could be either infected or coexist with infective endocarditis (IE). Both conditions present with identical clinical features and increased embolization risk, therefore instituting the need for prompt surgical resection [Yuan 2015; Lee 2017; Chang 2011]. We present a rare case of RV myxoma that was found to be complicated with concomitant tricuspid valve (TV) endocarditis during surgery.

\section{CASE REPORT}

A 53-year-old female presented with worsening dyspnea on exertion for 1 month. She also had intermittent fever and

Received Fuly 14, 2020; received in revised form August 4, 2020; accepted August 4, 2020.

Correspondence: Fenni Pratita, MD, Falan Fatinegara Timur 2/25, Jakarta Timur, DKI Jakarta, Indonesia; +6281283603537 (e-mail: jenni_pratita@ hotmail.com). decreased appetite for 4 months. She had a history of type-2 diabetes mellitus with no family history of myxoma. Physical exam was normal except for fever $\left(38.1^{\circ} \mathrm{C}\right)$ and poor oxygen saturation (92\% on $3 \mathrm{~L} / \mathrm{min}$ oxygen).

Laboratory examination showed anemia $(10.4 \mathrm{~g} / \mathrm{dL})$ and leukocytosis $\left(23,130 / \mathrm{mm}^{3}\right)$ with neutrophilia. Both erythrocyte sedimentation rate and procalcitonin were markedly increased $(91 \mathrm{~mm} / \mathrm{h}$ and $4.99 \mathrm{ng} / \mathrm{mL}$, respectively).

Transthoracic echocardiography showed a huge mobile mass attached to the interventricular septum beneath the TV and extending into the right atrium (Figure 1). Abdominal ultrasonography and chest X-ray showed no sign of metastasis.

She underwent surgery 4 days after admission. Empiric antibiotics and thrombocyte concentrate were administered preoperatively. The used approach was standard median sternotomy. Cardiopulmonary bypass (CPB) was established through aortic and bicaval cannulation and then aortic cross clamping was performed. Afterwards, right atriotomy was performed, showing a rounded $5 \times 4 \times 3 \mathrm{~cm}$ pedunculated mass. Unexpectedly, upon complete excision of the tumor, tiny masses $(<5 \mathrm{~mm})$ were found on the TV which, based on the color and consistency, were considered as vegetations (Figure 2).

Normal saline test showed TV incompetence, so DeVega annuloplasty was performed. A double continuous suture was performed along the anterior and posterior annulus using 3/0 polypropylene suture, while the septal portion is spared to protect the conduction system. The suture was commenced at the posterior part of the annulus' septal portion and continued in anticlockwise direction, with the needle penetrating at 1-2 $\mathrm{mm}$ depth in 5-6 $\mathrm{mm}$ long bites until the fibrous trigone was reached. Then it was reversed over a pledget. Each bite of the second suture line intercalated that of the first one and the suture was finished at the starting point where it was tied over a pledget. The degree of the narrowing of the annulus was controlled using a Hegar dilator [Antunes 2003].

Serial blood and vegetation cultures were both negative. Pathologic examination of the mass was suggestive of myxoma, showing wide myxoid area with oval/stellate cells containing pleomorphic nuclei, fine chromatin, and eosinophilic cytoplasm (Figure 2).

The postoperative period was uneventful. The patient was treated with antibiotics (Ceftriaxone) for six weeks. Followup transthoracic echocardiography after one month showed no residual mass with trivial tricuspid regurgitation. 


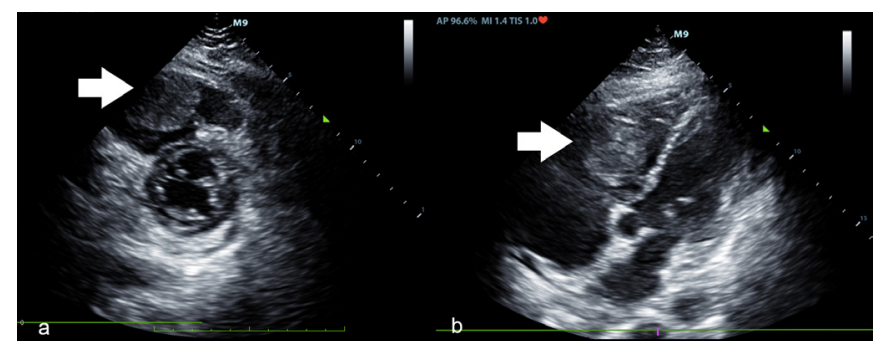

Figure 1. Echocardiography showing a mobile mass $(A)$ with visible stalk attached to interventricular septum (B).

\section{DISCUSSION}

Cardiac tumors are very rare, with around $75 \%$ of them being benign in origin. Although myxoma is the most common form of benign cardiac tumors, less than $3-4 \%$ of cases are detected in the RV. In accordance with our patient, it is more common among older adults with female predilection. Most cases, including ours, are sporadic [Koza 2019].

The clinical presentation of myxomas depends on its size and location. Dyspnea, as reported by our patient, is the most common symptom [Lee 2017]. Specifically, RV myxomas may cause symptoms of right heart failure due to obstruction of RV inflow and/or outflow tract [Koza 2019].

Myxomas can mimic systemic autoimmune diseases through secretion of interleukin 6 and 8. Therefore, systemic symptoms, such as fever and decreased appetite found on our patient, are found in $80-85 \%$ of cases [David 2018].

In our case, fever was accompanied by marked leukocytosis and increased inflammatory markers, raising the suspicion for infection. Infected myxomas are very rare with only 39 cases reported between 1998-2014. Among these cases, fever is the most common symptom [Yuan 2015]. However, according to the clinical criteria proposed by Revankar and Clark, our case did not qualify as infected cardiac myxoma, as both blood culture and inflammation on pathology were negative [Revankar 1998].

Intraoperative findings of vegetations on the $\mathrm{TV}$, together with TV incompetence, suggested concomitant TV endocarditis. We failed to detect the vegetations on preoperative transthoracic echocardiography, which reportedly only has a sensitivity of $21 \%$ in detecting IE. On the contrary, transesophageal echocardiography has $86 \%$ sensitivity and therefore should be preferred [Sekar 2017]. RV myxoma, especially with concurrent IE, is very rare and has identical clinical features with infected myxoma. In this case, both serial blood and vegetation cultures were negative, probably due to previous antibiotics administration.

The right atrial surgical approach is sufficient in most RV myxoma cases [Gajjar 2011]. Cardiac myxomas, especially infected ones, are prone to embolism. Similarly, IE could also induce embolism. Therefore, preventive measures should be taken during surgery [Yuan 2015; Chang 2011]. These include prevention of inconvenient manipulation of the tumor and heart, especially before aortic cross-clamping, and looping of

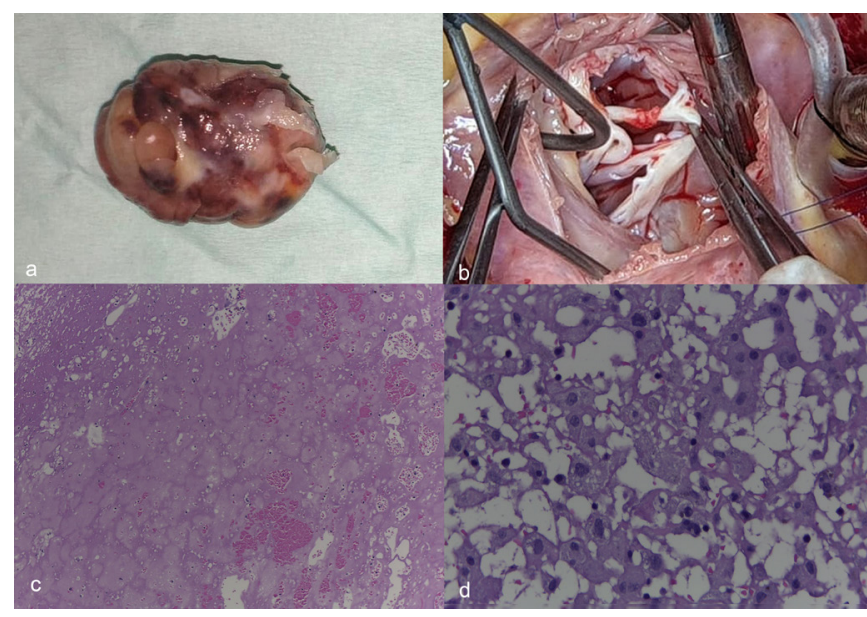

Figure 2. The resected myxoma (A) and vegetations on tricuspid valve (B). Pathology examination showed myxoid area (C) and macrophages (D).

both vena cava afterward [Gajjar 2011].

In this patient, although vegetations were found, there was no significant leaflet pathology and the TV incompetence was mainly due to the dilated annulus caused by the mass. Therefore, we decided to perform DeVega annulopasty [Antunes 2003]. DeVega has been reported as a safe and effective method to treat TV incompetence, with a 10 -year freedom rate of more than $90 \%$, especially when the annulus is reduced appropriately considering patients' body size using cylindrical sizers (i.e. Hegar dilators) [Hwang 2013].

In accordance with typical myxoma characteristics, our patient's mass was pedunculated with yellowish color and a smooth surface. The size of our patient's mass $(5 \times 4 \times 3$ $\mathrm{cm}$ ) was rather big, as myxomas range from $1-10 \mathrm{~cm}$ in size [Lee 2017]. Although recurrences rarely occur in sporadic cases, regular echocardiogram should be continued [Koza 2019].

\section{REFERENCES}

Antunes MJ. 2003. De Vega annuloplasty of the tricuspid valve. Oper Tech Thorac Cardiovasc Surg. 8:169-76.

Chang JH, Kim JY, Yoon JW, et al. 2011. A case of infected left atrial myxoma with concomitant mitral valve endocarditis. Korean Circ J 41:618-21.

David W, Rehman N, Singh R, David S. 2018. Inverted yoga and near syncope: an unusual diagnosis of right ventricular myxoma. Case Reports Cardiol 2018:1-4.

Gajjar TP, Shah GB, Desai NB. 2011. Giant ventricular myxoma obstructing right ventricular outflow tract. Brazilian J Cardiovasc Surg 26:663-6.

Hwang HY, Chang HW, Jeong DS, Ahn H. 2013. De vega annuloplasty for functional tricupsid regurgitation: Concept of tricuspid valve orifice index to optimize tricuspid valve annular reduction. J Korean Med Sci 28:1756-61. 
Koza Y, Arslan U, Erkut B, Koza E. 2019. Giant right ventricular mass protruding into the pulmonary artery during systole. J Cardiovasc Echogr 29:68.

Lee KS, Kim GS, Jung Y, et al. 2017. Surgical resection of cardiac myxoma-a 30-year single institutional experience. J Cardiothorac Surg 12:1-6.

Revankar SG, Clark RA. 1998. Infected cardiac myxoma case report and literature review. Med 77:337-44.
Sekar P, Johnson JR, Thurn JR, et al. 2017. Comparative sensitivity of transthoracic and transesophageal echocardiography in diagnosis of infective endocarditis among veterans with Staphylococcus aureus bacteremia. Open Forum Infect Dis 4:1-8.

Yuan S. 2015. Infected Cardiac Myxoma: an Updated Review. Braz J Cardiovasc Surg 30:571-8. 